\title{
Adaptations and Deficits in the Vestibulo-Ocular Reflex after Peripheral Ocular Motor Palsies
}

\author{
JAMES A. SHARPE, ${ }^{a, b}$ DOUGLAS TWEED, ${ }^{a, c}$ AND AGNES M.F. WONG ${ }^{a, b}$ \\ ${ }^{a}$ Division of Neurology, and Departments of ${ }^{b}$ Ophthalmology, and ${ }^{c}$ Physiology, \\ University Health Network, University of Toronto, Toronto, Ontario, Canada
}

\begin{abstract}
Palsy of a nerve might be expected to lower vestibulo-ocular reflex (VOR) responses in its fields of motion, but effects of peripheral neuromuscular disease were unknown. We recorded the VOR during sinusoidal head rotations in yaw, pitch, and roll at $0.5-2 \mathrm{~Hz}$ and static torsional gain in 43 patients with unilateral nerve palsies. Sixth nerve palsy $(n=21)$ reduced both abduction and adduction VOR gains in darkness. In light, horizontal visually enhanced VOR (VVOR) gains were normal in moderate and mild palsy. In severe palsy, horizontal VVOR gains remained low in the paretic eye when it was fixating, whereas gains in the nonparetic eye became higher than normal. Third nerve palsy $(n=10)$ decreased VOR and VVOR gains during abduction, adduction, elevation, depression, extorsion, and intorsion. Fourth nerve palsy $(n=13)$ reduced VOR gains of the paretic eye during intorsion, extorsion, elevation, depression, abduction, and adduction, but in light vertical and horizontal VVOR gains were normal. In the nonparetic eye, all gains were normal. Reduced VOR gains in the direction of paretic muscles and also in the direction of their antagonists, together with normal gains in the nonparetic eye, indicate a selective adjustment to the antagonists of paretic muscles. Increase of VVOR gains to normal in the paretic eye, when used for fixation, without conjugate increase in gains in the occluded nonparetic eye, provides further evidence of selective adaptation for the paretic eye. Motions of the eyes after nerve palsies indicate monocular VOR adaptation in three dimensions.
\end{abstract}

KEYWORDS: adaptation; nerve palsies; sixth nerve palsy; third nerve palsy; fourth nerve palsy; static torsional vestibulo-ocular reflex; angular vestibuloocular reflex; torsional vestibulo-ocular reflex; vertical vestibulo-ocular reflex; horizontal vestibulo-ocular reflex; Hering's law; diplopia

\section{INTRODUCTION}

Assessment of strabismus emphasizes static deviations and little information is available about the effects of paralytic strabismus on eye-movement dynamics such as during the vestibulo-ocular reflex (VOR). Adaptive changes in the VOR occur in

Address for correspondence: Dr. James A. Sharpe, Division of Neurology, University Health Network TWH, WW 5-440, 399 Bathurst Street, Toronto, Ontario M5T 2S8, Canada. Fax: 416603-5596.

sharpej@uhnres.utoronto.ca

Ann. N.Y. Acad. Sci. 1004: 111-121 (2003). @ 2003 New York Academy of Sciences. doi: 10.1196/annals.1303.070 
response to different visual stimuli. ${ }^{1,2}$ Disconjugate VOR adaptation has been elicited in monkeys in response to anisometropic prisms ${ }^{3}$ and experimental weakening of the horizontal rectus muscles. ${ }^{4,5} \mathrm{We}$ examined the angular VOR in patients with unilateral peripheral sixth, third, and fourth nerve palsies to determine effects of palsies in different directions on the VOR and its adaptation, if any, in each eye to monocular palsies. We identified changes in the actions of antagonists to paretic muscles that indicate monocular adaptations to peripheral neuromuscular deficits.

\section{METHODS}

Patients with unilateral peripheral palsy of the sixth $(n=21)$, third $(n=10)$, or fourth $(n=13)$ ocular motor nerves were recruited from the Neuroophthalmology Center at the University Health Network. ${ }^{6-8}$ The duration and age of onset of diplopia, the presence or absence of risk factors for ischemia (diabetes mellitus and hypertension), and associated neurologic symptoms and signs were determined. The magnitude of strabismus was measured objectively using the prism and cover test and subjectively using the Maddox rod and prism test. Appropriate tests were performed to rule out myasthenia gravis, thyroid ophthalmopathy, other orbital diseases, or intracranial lesions. Ranges of duction were estimated as the estimated percentage of the normal abduction in the other eye. On the basis of the duction defect, patients with sixth nerve palsies were classified into three groups: mild (81$95 \%$ of normal range of abduction), moderate (51-80\%), and severe ( $\leq 50 \%)$. Fifteen normal subjects of similar ages served as controls (mean age, 52 years; SD, 15 years; median age, 58 years; age range 19-69; 8 women).

The angular dynamic VOR was measured in darkness while patients made active sinusoidal head on body rotations in yaw and pitch at approximately 0.5 and $2 \mathrm{~Hz}$, and in roll at approximately $0.5,1$, and $2 \mathrm{~Hz}$ at amplitudes of approximately $\pm 10^{\circ}$ from orbital midposition. The dynamic visually enhanced VOR (VVOR) was measured while they fixated on a stationary laser target $1 \mathrm{M}$ from the naision, with one eye occluded, fixating in turn with the paretic and nonparetic eye. To measure the static torsional VVOR, we had patients fixate on the center target with one eye occluded as we measured their ocular responses to static head rolls of approximately $30^{\circ}$ toward each shoulder, as measured with a head search coil. Static torsional gain is defined as change in torsional eye position divided by change in head position during maintained head roll. The test then was repeated with the other eye fixating and the fellow eye occluded, and also in total darkness (VOR).

Eye movements were recorded by three-dimensional binocular magnetic scleral search coils (Skalar Instrumentation, Delft, the Netherlands) using 1.83-m-diameter field coils arranged in a cube (CNC Engineering, Seattle, WA). There was minimal cross talk; large horizontal and vertical movements produced deflections in the torsional channel of less than $4 \%$ of the amplitude of the horizontal and vertical movement. Any coil slippage was assessed by offsets in torsional eye position signal during testing. Consistency of calibrated positions after each eye movement provided evidence that the coil did not slip on the eye. Eye and head position signals were filtered with a bandwidth of 0 to $90 \mathrm{~Hz}$, and digitized at $200 \mathrm{~Hz}$.

Fast phases of vestibular nystagmus were identified ${ }^{9}$ and positions between $80 \mathrm{~ms}$ before and after fast phases were removed, the gaps being replaced with quadratic 
fits. The offset due to the fast phase then was removed, and the ongoing slow phase was interpolated to yield a cumulative trace of eye position.

Using a least squares sinusoidal fit, ${ }^{10}$ we fitted eye and head positions with one cycle, and phases and amplitudes and amplitude gains were computed. We also plotted head velocity against eye velocity and performed a linear regression for each direction. The slopes of the fitted lines were the gains, and the results were comparable to those computed by the least squares sinusoidal fit technique.

\section{RESULTS}

Of 21 patients with sixth nerve palsy, six had severe, seven had moderate, and eight had mild palsy; the duration of symptoms ranged from 2 weeks to 96 months (mean, 16 months). For 10 patients with third nerve palsies, the duration of symptoms ranged from 1 week to 50 months (mean, 18 months). In 13 patients with fourth nerve palsies, duration of symptoms ranged from 1 week to 132 months (mean, 35 months). VOR function was tested at one point in the course of their palsies and expressed as changes from normal, rather than serial intrasubject changes. Any recovery toward normal values was not assessed. Abnormalities are interpreted as deficits or adaptation to those deficits.

\section{Horizontal VOR}

Sixth nerve palsy. In all patients, horizontal VOR gains in darkness were decreased in the paretic eye in both abduction and adduction and remained normal in the nonparetic eye in both directions (FIG. 1). In light, horizontal VVOR gains were normal in both eyes in moderate and mild palsy. During active head movement, normal persons have both VOR (in darkness) and the VVOR gains approximating unity. ${ }^{11}$ In severe palsy, horizontal VVOR gains were low in the paretic eye in both directions, during viewing with either eye, whereas those in the nonparetic eye were higher than normal (>1.0) when the paretic eye viewed (FIG. 1). In light and darkness, mean phase differences between the eye and head positions approximated $180^{\circ}$, designated as zero phase shift.

Third nerve palsy. Horizontal VOR and VVOR gains of the paretic eye were decreased during both abduction and adduction.

Fourth nerve palsy. In darkness, horizontal VOR gains of the paretic eye were reduced symmetrically during both abduction and adduction $(P<0.01)$, whereas gains of the nonparetic eye were normal. In light, during paretic or nonparetic eye viewing, horizontal VVOR gains of both the paretic and the nonparetic eyes were normal $(P<$ $0.05)$. Neither eye showed any significant phase shift from zero in light or in darkness.

\section{Vertical VOR}

Sixth nerve palsy. Vertical VOR and VVOR gains were normal in severe and mild palsies.

Third nerve palsy. In darkness (FIG. 2, top graph), vertical VOR gains of the paretic eye were reduced $(P<0.01)$ symmetrically during both elevation and depression, whereas gains of the nonparetic eye were normal. In light, during paretic eye or nonparetic eye viewing (FIG. 2, middle and bottom graphs), vertical VVOR gains of the 


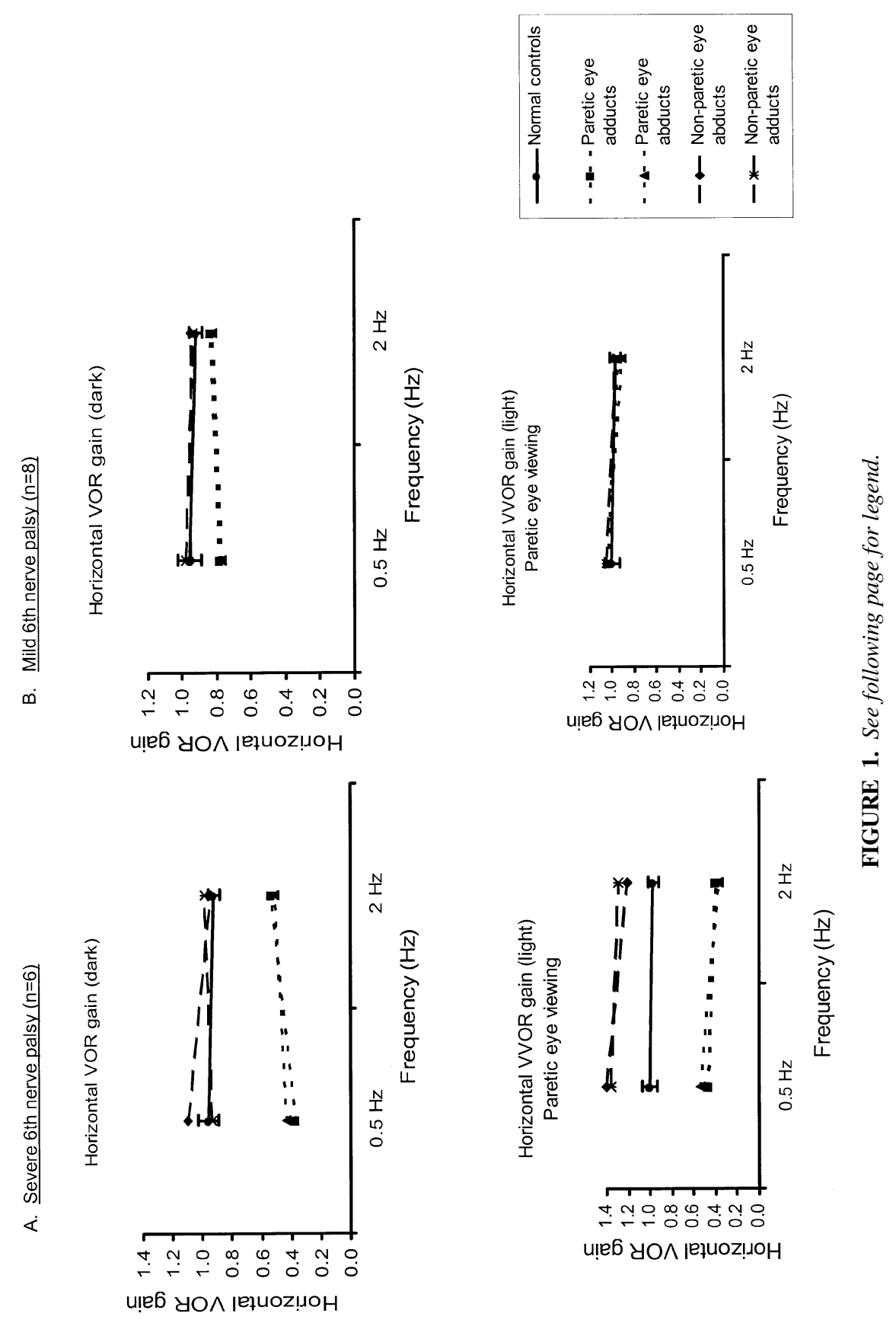


Vertical VOR gain (dark)

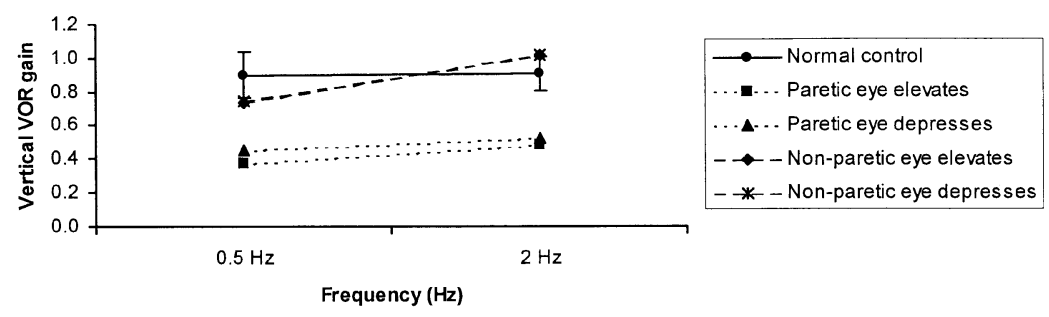

Vertical WOR gain (light) - Paretic eye viewing

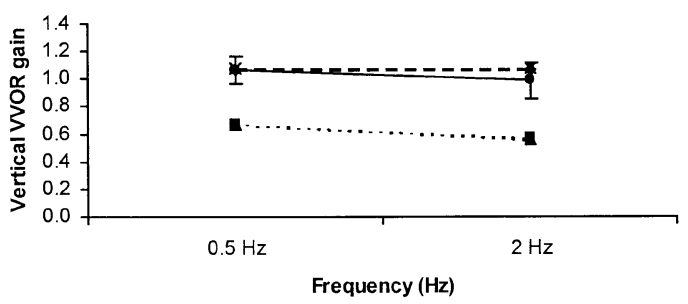

Vertical WVOR gain (light) - Non-paretic eye viewing

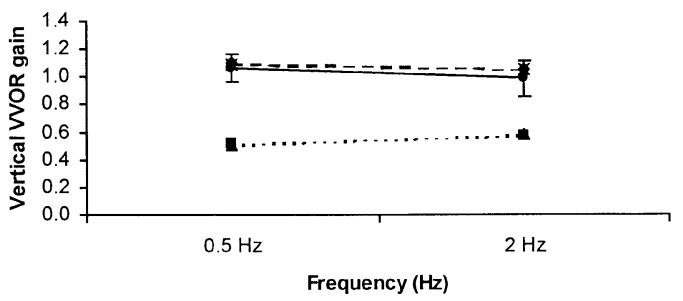

FIGURE 2. Vertical VOR and VVOR in 10 patients with third nerve palsy. The VOR gain of the paretic eye is severely reduced in elevation and depression (top). The VVOR gain remains low during fixation with the paretic eye or with the normal eye occluded. Error bars are 1 SD above and below group mean.

FIGURE 1. Horizontal VOR and VVOR gains during active head rotation at approximately 0.5 and $2 \mathrm{~Hz}$ in sixth nerve palsy. (A) Group mean VOR gains in severe palsy are reduced symmetrically in the paretic eye (top). VVOR gains with the paretic eye fixating and the normal eye occluded (bottom) become well above unity in the normal eye, but VVOR gains in the paretic eye remain reduced. (B) In mild palsy VOR gains for the patient group are reduced also in the paretic eye during abduction and adduction (top), but VVOR gains increase to normal values (bottom). Error bars indicate 1 SD. Insets in FIGURES 1, 2, and 4 show lines keys for paretic eye and normal eye and directions of movement. 
paretic eye remained reduced $(P<0.05)$, whereas gains in the nonparetic eye were normal. Neither eye showed any significant phase shift from zero in light or in darkness.

Fourth nerve palsy. In darkness, vertical VOR gains of the paretic eye were symmetrically reduced during both depression and elevation $(P<0.05)$, whereas gains of the nonparetic eye were normal. In light, during paretic eye and nonparetic eye viewing, vertical VVOR gains of both the paretic and the nonparetic eyes were normal $(P<0.05)$. Neither eye showed any significant phase shift from zero in light or in darkness.

\section{Torsional VOR, Dynamic and Static}

Sixth nerve palsy. Dynamic torsional VOR and VVOR gains were significantly reduced during head roll in both the paretic and nonparetic eyes when compared with normal controls $(P<0.05)$. Neither eye showed any significant phase shift from zero during vertical or torsional rotation. Static torsional gains were reduced in $19(90 \%)$ of the 21 patients in light and in dark (Z-tests, $P<0.05$ ). Static torsional VOR and VVOR gains of each eye were conjugate between the two eyes in all patients and did not differ during right eye or left eye viewing.

Third nerve palsy. In darkness dynamic torsional VOR gains of the paretic eye were reduced during both intorsion and extorsion $(P<0.01)$, whereas gains of the nonparetic eye were normal. In light, and during viewing with either eye, torsional VVOR gains of the paretic eye remained reduced $(P<0.01)$, whereas gains in the

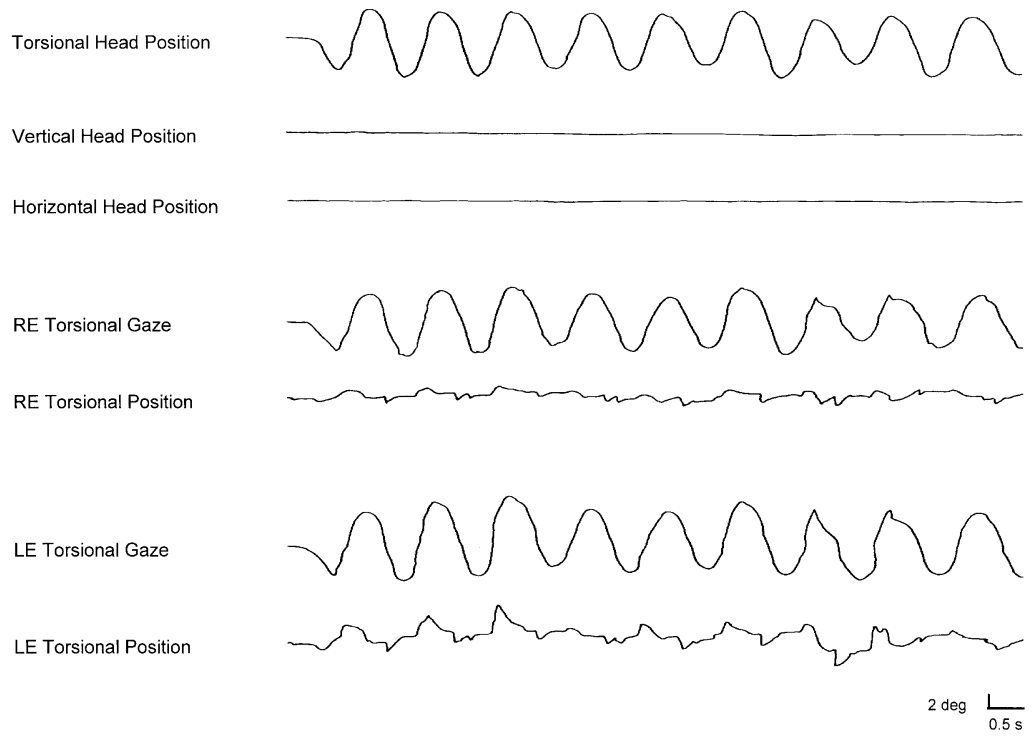

FIGURE 3. Torsional VOR in darkness during 2-Hz head roll in a patient with a right fourth nerve palsy. Responses are low in the right eye during both extortion and intorsion and normal in the left eye. Gaze is summed head and eye motion as recorded by a search coil. Upward movements in torsional traces are clockwise with reference to the patient (i.e., toward her right shoulder), and downward movements are counterclockwise. 
nonparetic eye were normal. ${ }^{7}$ In normal persons, viewing a foveal target does not appreciably raise torsional VVOR gains of above torsional gains in darkness. ${ }^{12}$ Neither eye showed any significant phase shift from zero in light or in darkness. Static torsional VOR and VVOR gains of the paretic eye were reduced during intorsion and extorsion $(P<0.05)$ but were normal in the nonparetic eye.

Fourth nerve palsy. In darkness, dynamic torsional VOR gains of the paretic eye were reduced symmetrically during intorsion and extorsion (FIG. 3) in each of 13 patients $(P<0.01)$, whereas gains of the nonparetic eye were normal (FIG. 4 , top graph). In light, during either paretic or nonparetic eye viewing (FIG. 4, middle and bottom graphs), torsional VVOR gains of the paretic eye were also low in both di-

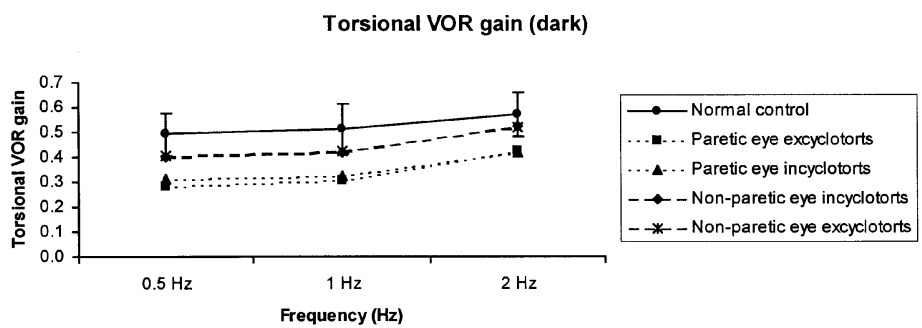

Torsional WOR gain (light) - Paretic eye viewing

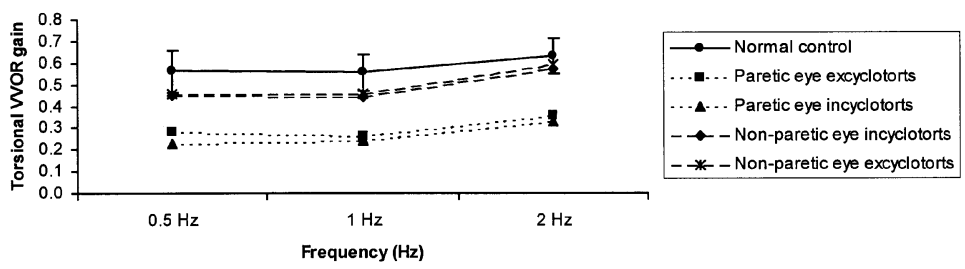

Torsional WOR gain (light) - Non-paretic eye viewing

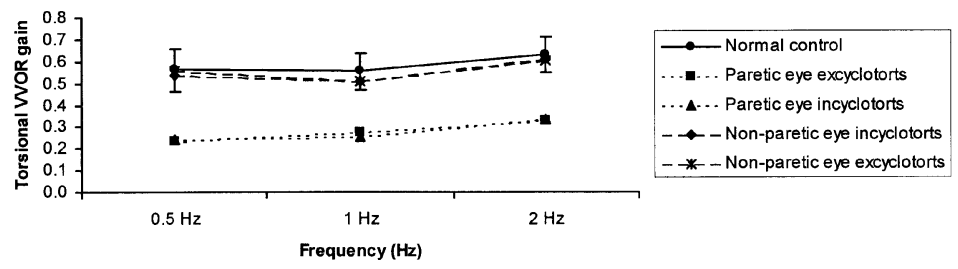

FIGURE 4. Torsional VOR and VVOR gains in 13 patients with trochlear nerve palsy. Intorsion and extortion gains are symmetrically reduced in the paretic eye in darkness (top) and during fixation with either eye. A foveal target has no appreciable effect on torsional gain. Error bars are 1 SD. 
rections $(P<0.05)$, whereas those of the nonparetic eye remained normal. ${ }^{8}$ In light and in darkness, the mean phase was zero. Static torsional VOR and VVOR gains of the paretic eye were reduced during intorsion $(P<0.05)$ and normal during extorsion of the paretic eye. In the nonparetic eye, static torsional VOR and VVOR gains were normal.

\section{DISCUSSION}

During head rotation in darkness, angular VOR gains are reduced during movement in the directions of actions of paretic muscles as anticipated from their palsy. However, dynamic gains are also reduced in the fields of actions of their antagonist muscles. VOR gains in the nonparetic eye remain normal, indicating a selective adjustment of the paretic eye, specifically to the antagonists of paretic muscles. In light, visual input increases gain of the paretic eye when it is used for fixation, providing further evidence of selective adaptation in the paretic eye. Torsional dynamic VOR and VVOR gains of the paretic eye are reduced for both extortion and intorsion in third and fourth nerve palsies. Motion of the eyes after nerve palsies exemplifies monocular adaptation of the VOR in three dimensions.

\section{Sixth Nerve Palsy}

In darkness, horizontal VOR gains are reduced during abduction of the paretic eye in all patients, as anticipated in sixth nerve palsy. Gains are also reduced during adduction of the paretic eye (FIG. 1), suggesting that innervation to the medial rectus has changed. After severe palsy, vision does not increase abducting or adducting horizontal VVOR gains to normal in the paretic eye but causes secondary increase in VVOR gains to values above unity in the nonparetic eye, when the paretic eye fixates. To adopt a conventional term from strabismology, this is a secondary change in the VOR, occurring when the paretic eye fixates. In mild and moderate palsy, vision enhances the VOR in the paretic eye but causes no change in the nonparetic eye, suggesting a monocular readjustment of innervation selectively to the paretic eye. Vertical VOR and VVOR gains are normal, indicating that the lateral rectus does not have significant vertical actions through the $\pm 10^{\circ}$ excursions that we tested.

Reduced torsional VOR gains in the paretic eye can be explained by the esotropia in sixth nerve palsy. Torsional VOR gain normally varies with vergence. ${ }^{13,14}$ We attribute the reduced torsional gains in the paretic eye to the mechanism that normally lowers it during convergence. ${ }^{6}$ The low torsional gains in the nonparetic eye may be an adaptation to reduce torsional disparity between the two eyes.

\section{Third Nerve Palsy}

Adducting VOR gains are reduced, as anticipated from medial rectus palsy. Abducting gains are also reduced; the reduction is attributed to an adaptive decrease in innervation to the lateral rectus, to achieve symmetry of the horizontal VOR. Torsional VOR gains are reduced during extorsion from palsy of the inferior oblique muscle. Gains are also reduced during intorsion, ${ }^{7}$ which may be explained by an adaptive decrease in innervation to the superior oblique, to restore symmetry of the torsional VOR. 


\section{Fourth Nerve Palsy}

During head rotation in darkness, VOR gains are reduced during intorsion, depression, and abduction of the paretic eye, as anticipated from paresis of the superior oblique muscle. VOR gains during extorsion (FIG. 3), elevation, and adduction of the paretic eye also are reduced, whereas VOR gains in the nonparetic eye remain normal, indicating a selective central adjustment of innervation to the paretic eye. In light, torsional VVOR gains in the paretic eye remained reduced (FIG. 4). Visual input increases vertical and horizontal VVOR gains to normal in the paretic eye, without a conjugate increase in VVOR gains in the nonparetic eye, ${ }^{8}$ providing further evidence of selective adaptation in the paretic eye.

Patients selected a preferred eye for viewing during the course of their palsies. We did not control the eye of habitual fixation, which was probably the nonparetic eye in most cases, although they may have switched from one eye to the other at their whim or subconscious choice. The adaptation might have differed if patients predominantly used the paretic eye for fixation.

\section{Changes in Antagonists of Paretic Agonist Muscles}

Without the decreased VOR gains in the direction of action of antagonists of paretic muscles, the VOR would be asymmetric in the paretic eye. The asymmetry would drive the paretic eye further into direction of action of the antagonist with each cycle of head rotation, resulting in increasing position disparity between the two eyes and more diplopia (FIG. 5). The brain might adopt any one of four strategies to prevent this disparity. First, it might increase its innervation to the paretic agonist to increase VOR gain of the paretic eye, but this strategy is limited by the palsy itself. Second, the brain might generate saccades in direction of paresis to correct for the low agonist VOR gains. However, during activation of the paretic agonist, if common premotor signals are sent to motoneurons of the yolked agonist in the other eye, unwanted saccades would appear conjugately in the nonparetic eye, driving its fovea off its target. Third, the brain might attempt to prevent asymmetry of the VOR by

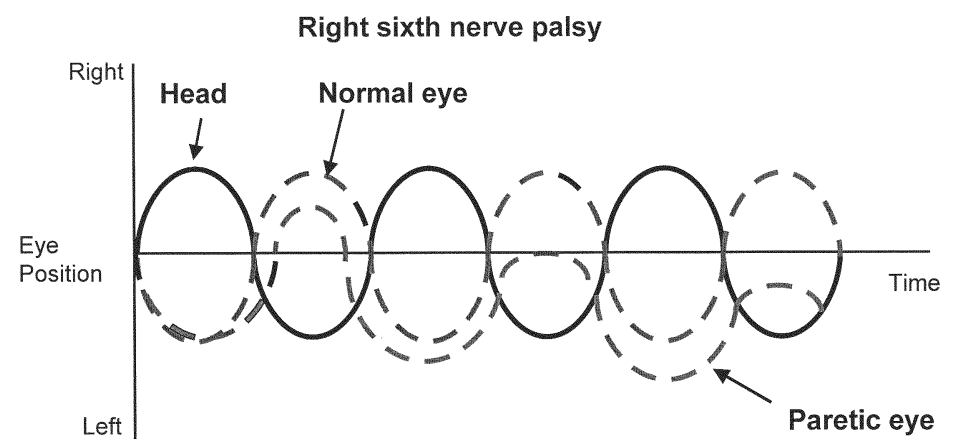

FIGURE 5. Schematic of consequence of asymmetry of VOR in unilateral sixth nerve palsy. Without adaptation to achieve symmetry, the paretic eye would move further into adduction with each cycle of head motion. 
decreasing antagonist gains in the paretic eye. Hering ${ }^{15}$ suggested that the brain controls gaze by two systems, one for conjugate movements and the other for vergence. However, if common and conjugate premotor signals were sent to motoneurons to both eyes, the yolked antagonist gain in the other (normal) eye would be reduced as well. For example, in the case of a left lateral rectus weakness from a left sixth nerve palsy, any adaptive reduction in innervation to the left medial rectus muscle would be accompanied by reduced innervation to the right lateral rectus muscle, in accord with Hering's proposal or "law." It was not. Fourth, the brain could selectively reduce VOR gains during action of the antagonist of the paretic muscle by reducing its innervation. This is apparently the strategy that the brain uses, in violation of Hering's law.

Changes in normal orbital plant mechanics might contribute to the decreased VOR gains of the paretic eye in the direction of the antagonists to paretic muscles. The relative contribution of agonist contraction and antagonist relaxation varies with orbital position, ${ }^{16}$ and it may be altered when one muscle of an agonist-antagonist pair is palsied. Contracture is characterized by muscle shortening and stiffening as a result of decreased number of sarcomeres. ${ }^{17}$ If the reduction of VOR gains in both directions were caused by changes in extraocular muscle mechanics, one would expect VOR gains to be subnormal during rotation or in light (VVOR) as well as in darkness, and that the peak velocities of nystagmus quick phases would be reduced in each direction. However, our results indicate that although VOR gains were decreased, VVOR gains could increase to normal values in light. In addition, although VOR gains were reduced in each direction, peak velocities of saccades in the antagonist's direction of action were normal (data not shown here). Our results provide evidence that decrease in VOR gains is not the result of changes in mechanical properties of the orbital plant but is caused by a functional central adaptation to the palsy.

Proprioceptive signals from extraocular muscles ${ }^{18}$ might contribute to VOR adaptation. Proprioceptive afferent fibers project via the ophthalmic branch of the trigeminal nerve to the spinal trigeminal nucleus, but a portion also may enter via the ocular motor nerves. ${ }^{19}$ Although visual information plays a massively dominant role in the control of VOR, altered proprioceptive inflow from a shortened (slack) antagonist or a palsied muscle might participate in the monocular adaptations that we identified after peripheral nerve palsies. Binocular disparity of retinal images that increases during head motion and asymmetry of retinal image slip when the VOR is imbalanced by palsy of a muscle appears to be the visual drive for monocular adaptation to reduce image slip and diplopia.

\section{ACKNOWLEDGMENTS}

This work was supported by Canadian Institutes of Health Research grants MT 15362 and ME 5504.

\section{REFERENCES}

1. Gonshor, A. \& G. Melvill Jones. 1976. Extreme vestibulo-ocular adaptation induced by prolonged optical reversal of vision. J. Physiol. (Lond.) 256: 381-414. 
2. Yagi, T., M. Shimizu, S. Sekine \& T. Kamio. 1981.New neurootological test for detecting cerebellar dysfunction. Vestibulo-ocular reflex changes with horizontal vision-reversal prisms. Ann. Otol. Rhinol. Laryngol. 90: 276-280.

3. OOHIRA, A. \& D.S. ZEE. 1992. Disconjugate ocular motor adaptation in rhesus monkey. Vision Res. 32: 489-497.

4. SNOw, R., J. HoRE \& T. VILIS. 1985. Adaptation of saccadic and vestibulo-ocular systems after extraocular muscle tenectomy. Invest. Ophthalmol. Visual Sci. 26: 924 931.

5. VIRRE, E., C. WERnER \& T. VILIS. 1988. Monocular adaptation of the saccadic system and vestibulo-ocular reflex. Invest. Ophthalmol. Visual Sci. 29: 1339-1347.

6. WONG, A.M.F., D. TwEED \& J.A. SHARPE. 2002. Adaptations and deficits in the vestibulo-ocular reflex after sixth nerve palsy. Invest. Ophthalmol. Visual Sci. 43: 99-111.

7. WONG, A.M.F. \& J.A. ShARPE. 2002. Adaptations and deficits in the vestibulo-ocular reflex after third nerve palsy. Arch. Ophthalmol. 120: 360-368.

8. WONG, A.M.F., J.A. SHARPE \& D. TwEED. 2002. The vestibulo-ocular reflex in fourth nerve palsy: deficits and adaptations. Vision Res. 42: 2205-2218.

9. RANALLI, P.J. \& J.A. SHARPE. 1988. Vertical vestibulo-ocular reflex, smooth pursuit and eye-head tracking dysfunction in internuclear ophthalmoplegia. Brain 111: 1299-1317.

10. SoKOLNIKOFF, I.S. \& E.S. SoKOLNIKOFF. 1941. Higher Mathematics for Engineers and Physicists. McGraw Hill. New York.

11. KIM, J.S. \& J.A. SHARPE. 2001. The vertical vestibulo-ocular reflex and its interaction with vision during active head motion: effects of aging. J. Vestib. Res. 11: 3-12.

12. MorRow, M.J. \& J.A. SharPE. 1993. The effects of head and trunk position on torsional vestibular and optokinetic eye movements in humans. Exp. Brain Res. 95: 144-150.

13. TweED, D., M. FeTter, D. Sievering, et al. 1994. Rotational kinematics of the human vestibuloocular reflex. II. Velocity steps. J. Neurophysiol. 72: 2480-2489.

14. Misslisch, H., D. TweED \& B.J.M. HESS. 2001. Stereopsis outweighs gravity in the control of the eyes. J. Neurosci. 21: RC126.

15. HeRING, E. 1868. Die Lehre vom binokularen Sehen. Wilhelm Englemann. Leipzig.

16. Collins, C.C. 1975. The human ocular system. In Basic Mechanisms of Ocular Motility and their Clinical Implications. G. Lennerstrand \& P. Bach-y-Rita, Eds.: 145180. Pergamon Press, New York.

17. ScotT, A.B. 1994. Change of eye muscle sarcomeres according to eye position. J. Pediatr. Ophthalmol. Strabismus 31: 85-88.

18. HAYMAN, M.R., J.P. DONALDSON \& I.M. DONALDSON. 1995. The primary afferent pathway of extraocular muscle proprioception in the pigeon. Neuroscience 69: 671-683.

19. GeNTLE, A. \& G. RuSKELL. 1997. Pathway of the primary afferent nerve fibers serving proprioception in monkey extraocular muscles. Ophthalmic Physiol. Opt. 17: 225231. 